\title{
Early treatment volume reduction rate as a prognostic factor in patients treated with chemoradiotherapy for limited stage small cell lung cancer
}

\author{
Joohwan Lee, MD¹, Jeongshim Lee, MD¹, Jinhyun Choi, MD², Jun Won Kim, MD², \\ Jaeho Cho, MD, PhD', Chang Geol Lee, MD, PhD' \\ 'Department of Radiation Oncology, Yonsei Cancer Center, Yonsei University College of Medicine, Seoul; \\ ${ }^{2}$ Department of Radiation Oncology, Gangnam Severance Hospital, Yonsei University College of Medicine, Seoul, Korea
}

Purpose: To investigate the relationship between early treatment response to definitive chemoradiotherapy (CRT) and survival outcome in patients with limited stage small cell lung cancer (LS-SCLC).

Materials and Methods: We retrospectively reviewed 47 patients with LS-SCLC who received definitive CRT between January 2009 and December 2012. Patients were treated with systemic chemotherapy regimen of etoposide/carboplatin $(n=15)$ or etoposide/cisplatin $(n=32)$ and concurrent thoracic radiotherapy at a median dose of 54 Gy (range, 46 to 64 Gy). Early treatment volume reduction rate (ETVRR) was defined as the percentage change in gross tumor volume between diagnostic computed tomography (CT) and simulation CT for adaptive RT planning and was used as a parameter for early treatment response. The median dose at adaptive RT planning was $36 \mathrm{~Gy}$ (range, 30 to $43 \mathrm{~Gy}$ ), and adaptive CT was performed in 30 patients (63.8\%).

Results: With a median follow-up of 27.7 months (range, 5.9 to 75.8 months), the 2-year locoregional progression-free survival (LRPFS) and overall survival (OS) rates were $74.2 \%$ and $56.5 \%$, respectively. The mean diagnostic and adaptive gross tumor volumes were $117.9 \mathrm{~mL}$ (range, 5.9 to $447 \mathrm{~mL}$ ) and $36.8 \mathrm{~mL}$ (range, 0.3 to $230.6 \mathrm{~mL}$ ), respectively. The median ETVRR was $71.4 \%$ (range, 30 to $97.6 \%)$ and the ETVRR $>45 \%$ group showed significantly better OS $(p<0.0001)$ and LRPFS $(p=0.009)$ than the other group.

Conclusion: ETVRR as a parameter for early treatment response may be a useful prognostic factor to predict treatment outcome in LS-SCLC patients treated with CRT.

Keywords: Small cell lung carcinoma, Radiotherapy, Tumor burden

\section{Introduction}

Small cell lung cancer (SCLC) is a well-known subtype of lung cancer that is diagnosed almost exclusively in patients with a smoking history [1]. SCLC comprised 13\% of new lung cancer diagnoses in 2011, and limited stage (LS)-SCLC accounts for approximately $30 \%$ to $40 \%$ of these cases in the United
States [2]. The current standard treatment for SCLC is systemic chemotherapy with concurrent thoracic radiotherapy (RT) for consolidation. While this cancer shows favorable response to treatment, it still has poor survival outcome $[3,4]$.

Prognostic factors for survival in SCLC include stage, age, sex, the World Health Organization performance score, serum lactate dehydrogenase level, N3 nodal status, and time from

Received 6 May 2015, Revised 3 June 2015, Accepted 10 June 2015.

Correspondence: Chang Geol Lee, MD, PhD, Department of Radiation Oncology, Yonsei Cancer Center, Yonsei University College Medicine, 50 Yonsei-ro, Seodaemun-gu, Seoul 120-752, Korea. Tel: +82-2-2228-8114, Fax: +82-2-312-9033, E-mail: CGLEE1023@yuhs.ac

(C) This is an Open Access article distributed under the terms of the Creative Commons Attribution Non-Commercial License (http://creativecommons.org/ licenses/by-nc/4.0/) which permits unrestricted non-commercial use, distribution, and reproduction in any medium, provided the original work is properly cited.

www.e-roj.org 
the start of any treatment to the end of radiation therapy (SER) [5]. The T stage is a significant prognostic factor for lung cancer, but it contains only bi-dimensional measurements of gross tumor. The International Commission on Radiation Units \& Measurements (ICRU) 50 report [6] defines gross tumor volume (GTV) as the gross palpable or visible/demonstrable extent and location of malignant growth and is the basic concept of three-dimensional conformal radiation therapy (3DCRT). Initial GTV is a prognostic factor for non-small cell lung cancer (NSCLC) patients treated with chemoradiotherapy (CRT) [7] and has been studied as an independent prognostic factor for survival in SCLC [8].

The volume reduction rate (VRR) during $R T$ is a possible indicator of biological tumor radiosensitivity and serves as a clinical marker of treatment outcome. In advanced rectal cancer patients treated with neoadjuvant CRT, Kim et al. [9] revealed a difference in tumor VRR between patients who were down-staged by neoadjuvant treatment and those who were not. Among head and neck (oropharyngeal or hypopharyngeal) cancer patients treated with definitive CRT using intensitymodulated radiation therapy (IMRT), VRR was a significant indicator of local control rate [10]. Werner-Wasik et al. [11] investigated the volumetric response of 22 locally-advanced NSCLC patients treated with non-surgical therapy including thoracic RT. VRR was one of the most effective tools for evaluating treatment response.

However, no studies investigate the prognostic effect of tumor volume change between initial presentation and adaptive RT planning on SCLC survival outcome. The aim of the current study is to investigate the relationship between treatment response and outcome in patients with LS-SCLC.

\section{Materials and Methods}

\section{Patients}

We retrospectively reviewed the medical records of 106 patients with SCLC who underwent CRT with definitive aim between January 2009 and December 2012. A diagnosis of SCLC was confirmed histologically in all patients. SCLC is staged by the Veterans Administration Lung Study Group (VALSG) staging system [12], according to which patients are classified as having limited disease or extensive disease. Patients with limited disease have tumor confined to the ipsilateral hemithorax, without any malignant effusion, with disease that may be incorporated in a single radiation beam field. Disease that does not fulfill these criteria is defined as extensive disease. Limited/extensive staging was determined based on imaging studies, including both diagnostic CT and positron emission tomography-computed tomography (PETCT). All eligible patients were also staged according to the 7th American Joint Committee on Cancer (AJCC) TNM staging system [13].

Exclusion criteria included extensive-stage disease ( $n=$ 19), more than one primary cancer that could affect survival outcome $(n=6)$, loss of follow-up after completion of treatment $(n=4)$, incomplete RT less than 45 Gy $(n=3)$, incomplete chemotherapy less than four cycles ( $n=13)$, and chemotherapy regimen other than etoposide/cisplatin (EP) or etoposide/carboplatin (EC) $(n=14)$. Finally, 47 patients were enrolled in the study.

\section{Pretreatment evaluation and follow-up}

Pretreatment evaluation included 1) complete history and physical examination, 2) biopsy for histological diagnosis, 3) laboratory studies, including complete blood cell counts and hepatic/renal functions, and 4) imaging studies for staging such as chest $X$-ray, chest $C T$, brain magnetic resonance imaging, and PET-CT. Patients were followed with chest CT and/ or PET-CT one month after radiotherapy, every three months within the first year, every six months in the second year, and once a year thereafter. Treatment response was evaluated with follow-up CT performed one and three months after RT using the Response Evaluation Criteria in Solid Tumors (RECIST) ver. 1.1 guidelines [14].

\section{Treatment}

1) Chemotherapy: To evaluate changes of tumor volume in response to $\mathrm{RT}$ evenly, we limited the concurrent chemotherapy regimen to only EP $(n=15)$ or EC $(n=32)$. The treatment schedule was consisted of cisplatin or carboplatin on day 1 with etoposide on days 1 to 3 for four to six cycles (average 4.3 cycles), and repeated every three weeks. The standard chemotherapy dose was $120 \mathrm{mg} / \mathrm{m}^{2}$ etoposide with $60 \mathrm{mg} / \mathrm{m}^{2}$ cisplatin for the EP regimen and $100 \mathrm{mg} / \mathrm{m}^{2}$ etoposide for the $\mathrm{EC}$ regimen. Carboplatin in the $\mathrm{EC}$ regimen dose was calculated based on the target area under the curve as $(5 \mathrm{mg} / \mathrm{mL} / \mathrm{hr} \times$ (glomerular filtration rate $[\mathrm{GFR}]+25)$ ). GFR was calculated with the Cockroft-Gault formula [15]. During treatment, six patients required a dose reduction due to hematologic toxicities more severe than RTOG grade 1 . There was a difference in volume between diagnostic CT and planning CT because RT started with the second cycle of chemotherapy in most patients $(n=35)$. 
2) Radiotherapy: All patients were treated with 3DCRT. Planning CT was performed with contrast and a 3-mm thickness for each scan slice. For respiration-dependent organ motion, fluoroscopy (Simulix Evolution; Nucletron, Veenendaal, the Netherlands) was used in 18 patients prior to July 2010, and a 4DCT system (Anzai Medical, Tokyo, Japan) that acquired images in 12 phases was used in 29 patients afterward that date.

For patients undergoing 2D fluoroscopy, GTV was defined as primary tumor and involved regional lymph nodes (LN) identified in the planning chest CT. The PET-CT was fused with the planning CT for target delineation in all but one patient (97.9\%). Clinical target volume (CTV) was defined as GTV plus a 10 to $15 \mathrm{~mm}$ margin in order to include microscopic tumor extension. Planning target volume (PTV), for adapting errors which could occur through set-up variation and respiratory motion, was defined as CTV plus an additional 5 to $15 \mathrm{~mm}$ margin, depending on 2D fluoroscopy results. For patients undergoing 4DCT, GTV was contoured on the mid-ventilation, maximum inhalation and exhalation phases in order to reflect respiration. CTV was defined as GTV plus a 10 to $15 \mathrm{~mm}$ margin expansion, and PTV was defined as an additional $5 \mathrm{~mm}$ margin beyond the CTV.

One patient was treated with IMRT, and 46 patients were treated with 3 DCRT. Three to five coplanar isocentric fields were designed for the initial 3DCRT plan, which used Pinnacle radiotherapy treatment planning systems (Philips Radiation Oncology Systems, Milpitas, CA, USA) or MIM software ver. 6.3.7 (MIM Software, Cleveland, OH, USA). An additional simulation CT was performed to evaluate GTV shrinkage during RT, and adaptive planning focused on accurate tumor targeting and preservation of adjacent normal organs (i.e., normal lung volume, esophagus). For this adaptive planning, we used a larger number of coplanar isocentric beams. In the patient treated with IMRT, the RT plan was designed to deliver 56 Gy to $95 \%$ of the PTV using six coplanar MV beams through a helical tomotherapy planning system. Definitive CRT was followed by prophylactic cranial irradiation (PCI) in patients showing complete or partial response.

\section{Volumetric parameters}

For each patient, we contoured GTVs in four sets of CT images: diagnostic, planning, adaptive, and follow-up CT images. 'Diagnostic CT' was defined as the first chest CT that contains a malignant lung mass confirmed by a chest radiologist. Chest CTs performed at other hospitals were also registered in our
Picture Archiving and Communication System (PACS) (GE Healthcare, Milwaukee, WI, USA) and fused with the 'planning CT' for target contouring. 'Adaptive CT' was defined as a chest simulation CT performed at the time of adaptive RT planning, using the same setting as the planning CT. 'Follow-up CT' was conducted for evaluation of the best treatment response between one and three months after completion of RT.

When contouring each GTV, we used MIM software ver. 6.3.7 and homogenized window settings for CT density in order to reduce errors. We defined a lung setting with a window of 1324 and a level of -362 for contouring GTV localized at the lung parenchyme and a mediastinum setting with a window of 500 and a level of 39 for regional LNs.

We proposed overall volume reduction rate (OVRR) as a parameter for overall treatment response that appeared as percentage change in GTV between diagnostic CT and followup CT. To evaluate early treatment response, we calculated early treatment volume reduction rate (ETVRR) as an indication of the percentage change in GTV obtained when comparing diagnostic CT with adaptive CT. Additionally, we measured early chemotherapy volume reduction rate (ECVRR) for pure evaluation of first cycle chemotherapy response defined as the percentage change in GTV between diagnostic CT and planning CT.

\section{Statistical analysis}

Locoregional progression-free survival (LRPFS), distant metastasisfree survival (DMFS), progression-free survival (PFS), and overall survival (OS) were calculated from the date of diagnosis to the event and were plotted using the Kaplan-Meier method. We dichotomized patients upon VRR with every 5\% interval around median to find out most effective cutoff value. For analyzing the relationship between the time interval from first chemotherapy to first RT and ETVRR, we used the Kruskal-Wallis test.

Univariate survival analyses for several prognostic factors were also processed with the Kaplan-Meier method. To create multivariate model, we used stepwise logistic regression with variables entry at $p<0.15$ and removed if the $p$-value was 20.15. Patient survival status was confirmed in February 2013. In our study, all statistical analyses were performed using SPSS ver. 20.0.0 (IBM SPSS, Armonk, NY, USA). A p-value $<0.05$ was considered statistically significant. 


\section{Results}

\section{Patient characteristics}

The median patient age was 68 years (range, 40 to 84 years), and 42 patients (89.4\%) were male. At the time of diagnosis, 41 patients (87.2\%) had the Eastern Cooperative Oncology Group performance status (ECOG PS) of $0(n=12)$ to $1(n=$ 29). According to the AJCC 7th TNM staging system [13], five patients were stage I (10.6\%), four patients were stage II (8.5\%) and 38 patients were stage III (80.9\%). The median thoracic RT dose was $54 \mathrm{~Gy}$ (range, 46 to $64 \mathrm{~Gy}$ ), with a median daily dose of 2 Gy (range, 1.8 to 2.5 Gy). The median time interval between diagnostic and planning CT was 32 days (range, 9 to 123 days). The date of first chemotherapy was a median of 22 days (range, 0 to 161 days) earlier than the date of first RT. Most patients started RT concurrently with $1 s t(n=8)$ or $2 n d$ $(n=35)$ cycle chemotherapy and other four patients received delayed RT because of poor general condition status $(n=2)$ or neutropenia caused by initial chemotherapy $(n=2)$.

Adaptive CT was conducted at a median RT dose of 36 Gy (range, 28.8 to $43 \mathrm{~Gy}$ ) and was performed in 30 patients (63.9\%). After completion of CRT, 38 patients (80.9\%) underwent $\mathrm{PCl}$. Patient characteristics are provided in Table 1.

\section{Treatment response and pattern of failure}

Five patients (10.6\%) achieved a complete response, 40 patients achieved a partial response (85.1\%), and two patients had stable disease (4.3\%). With a median follow-up time of 27.4 months (range, 6.2 to 66.4 months), the 2-year LRPFS and DMFS rates were $74.2 \%$ and $70.1 \%$, respectively. Twoyear PFS and OS rates were $50.6 \%$ and $56.5 \%$, respectively (Fig. 1). Of the total patients, 17 (36.2\%) experienced locoregional failure, 17 (36.2\%) had distant failure, and seven (14.9\%) had both. Sites of distant failure included brain $(n=9)$, bone ( $n$ $=2)$, adrenal gland $(n=2)$, liver $(n=2)$, and intra-abdominal LNs including the para-aortic area $(n=1)$. One patient had multifocal sites, including the brain, left seventh rib, and LN around the celiac trunk.

\section{Volumetric parameters related to survival outcome}

The volumetric parameters measured in this study are described in Table 2. The mean GTV of diagnostic, planning, adaptive, and follow-up CT were $117.9 \mathrm{~mL}$ (range, 5.9 to $447 \mathrm{~mL}$ ), $68.7 \mathrm{~mL}$ (range, 2.5 to $391 \mathrm{~mL}$ ), $36.8 \mathrm{~mL}$ (range, 0.3 to $230.6 \mathrm{~mL}$ ), and $20.6 \mathrm{~mL}$ (range, 0.1 to $102.1 \mathrm{~mL}$ ), respectively. Every patient experienced volume reduction in each CT when treated with CRT. The median OVRR was $81.6 \%$ (range, $44.2 \%$ to 99\%), median ECVRR was $53.5 \%$ (range, $0 \%$ to $82.3 \%$ ) and the median ETVRR of 30 patients with adaptive CT was $71.4 \%$ (range, $30 \%$ to $97.6 \%)$.

ETVRR was a statistically effective prognostic factor for OS $(p=0.012)$ and PFS $(p=0.015)$. In the univariate analysis

Table 1. Patient characteristics

\begin{tabular}{|c|c|}
\hline Characteristic & Value \\
\hline Age (yr) & $68(40-84)$ \\
\hline \multicolumn{2}{|l|}{ Sex } \\
\hline Male & $42(89.4)$ \\
\hline Female & $5(10.6)$ \\
\hline \multicolumn{2}{|l|}{ ECOG PS } \\
\hline 0 & $12(25.5)$ \\
\hline 1 & $29(61.7)$ \\
\hline 2 & $6(12.8)$ \\
\hline \multicolumn{2}{|l|}{ T stage } \\
\hline $1-2$ & $22(46.8)$ \\
\hline $3-4$ & $25(53.2)$ \\
\hline \multicolumn{2}{|l|}{$N$ stage } \\
\hline $0-2$ & $32(68.1)$ \\
\hline 3 & $15(31.9)$ \\
\hline \multicolumn{2}{|l|}{ AJCC stage } \\
\hline IA & $3(6.4)$ \\
\hline IB & $2(4.3)$ \\
\hline$\| A$ & $3(6.4)$ \\
\hline$\| B$ & $1(2.1)$ \\
\hline$\| \mathrm{A}$ & $15(31.9)$ \\
\hline$\| I I B$ & $23(48.9)$ \\
\hline \multicolumn{2}{|l|}{ Chemotherapy } \\
\hline \multicolumn{2}{|l|}{ Regimen } \\
\hline Etoposide/carboplatin & 15 (31.9) \\
\hline Etoposide/cisplatin & $32(68.1)$ \\
\hline \multicolumn{2}{|l|}{ Cycles } \\
\hline 4 & $39(83.0)$ \\
\hline 5 & $1(2.1)$ \\
\hline 6 & $7(14.9)$ \\
\hline \multicolumn{2}{|l|}{ Radiotherapy } \\
\hline \multicolumn{2}{|l|}{ Modality } \\
\hline 3DCRT & 46 (97.9) \\
\hline Tomotherapy & $1(2.1)$ \\
\hline Dose (Gy) & $54(46-64)$ \\
\hline \multicolumn{2}{|l|}{$\mathrm{PCl}$} \\
\hline Yes & $38(80.9)$ \\
\hline No & 9 (19.1) \\
\hline SER (day) & 64 (36-203) \\
\hline
\end{tabular}

Values are presented as median (range) or number of patients (\%). ECOG PS, Eastern Cooperative Oncology Group performance status; AJCC, American Joint Committee on Cancer; 3DCRT, threedimensional conformal radiation therapy; $\mathrm{PCl}$, prophylactic cranial irradiation; SER, start of any treatment to the end of radiation therapy. 
A

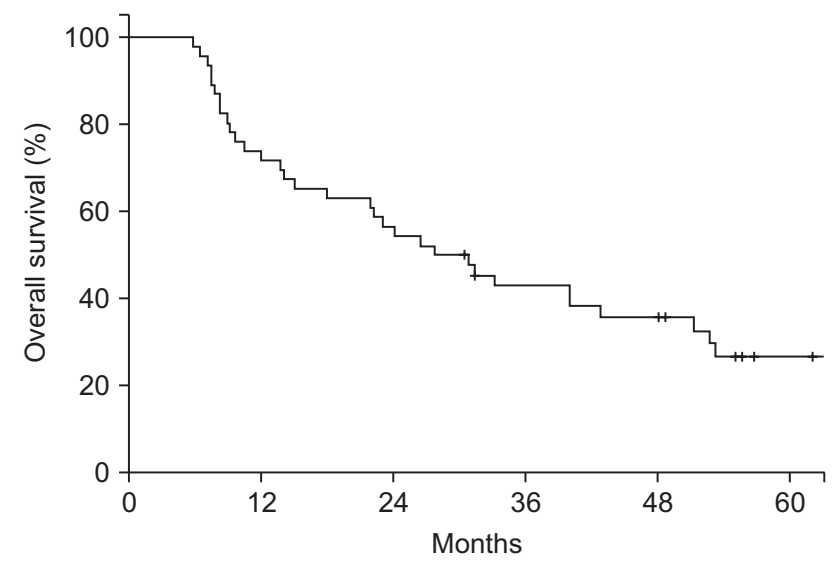

C

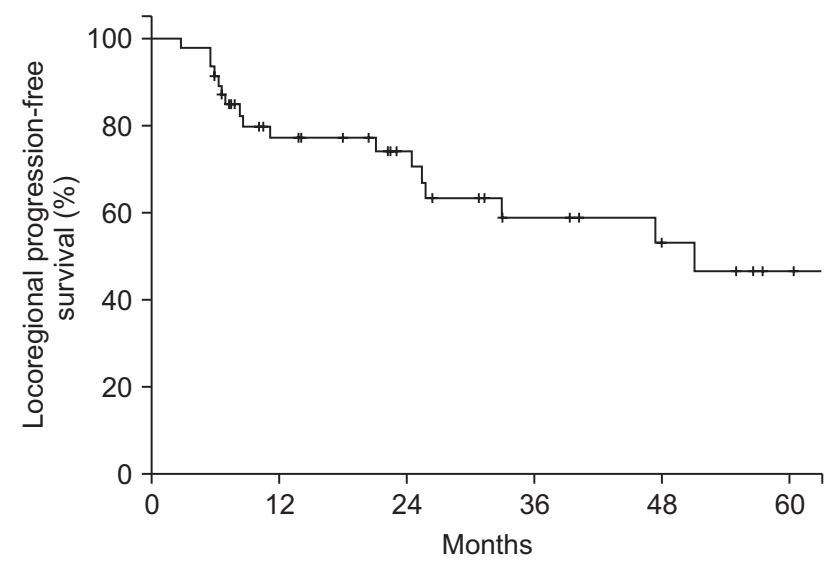

B

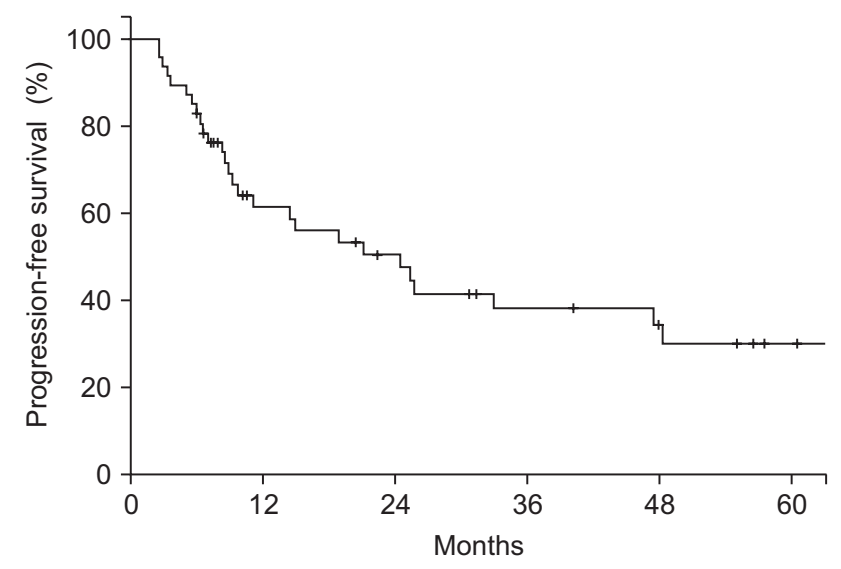

D

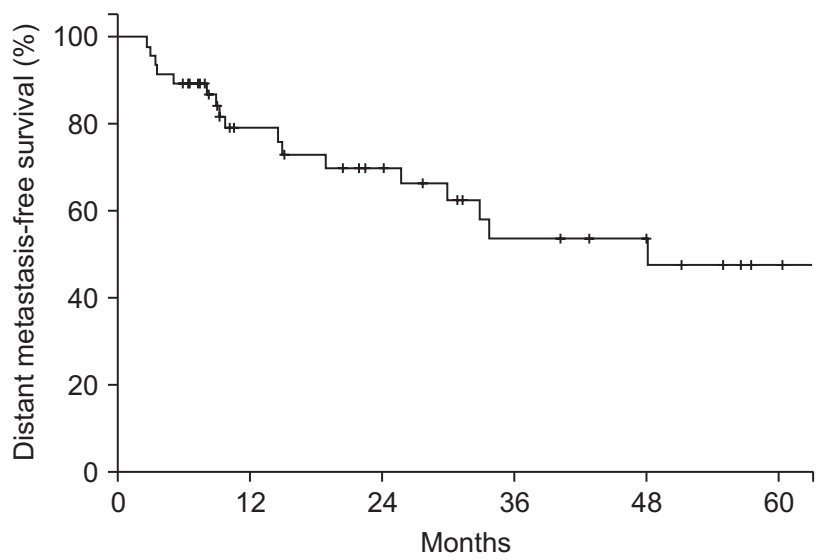

Fig. 1. Kaplan-Meier survival curves of 47 patients: (A) overall survival, (B) progression-free survival, (C) locoregional progression-free survival and (D) distant metastasis-free survival.

Table 2. Volumetric parameters

\begin{tabular}{cc}
\hline \multicolumn{1}{c}{ Parameter } & Value \\
\hline Gross tumor volume $(\mathrm{mL})$ & \\
Diagnostic CT $(\mathrm{n}=47)$ & $68.7(2.5-3-391)$ \\
Planning CT $(\mathrm{n}=47)$ & $36.8(0.3-230.6)$ \\
Adaptive CT $(\mathrm{n}=30)$ & $20.6(0.1-102.1)$ \\
Follow-up CT $(\mathrm{n}=47)$ & \\
Volume reduction rate $(\%)$ & $81.6 \pm 13.5(44.2-99)$ \\
Overall & $53.5 \pm 25.9(0-82.3)$ \\
Early chemotherapy & $71.4 \pm 16.6(30-97.6)$ \\
Early treatment &
\end{tabular}

Values are presented as mean (range) or median \pm standard deviation (range).

$\mathrm{CT}$, computed tomography.

with median cutoff value, initial GTV, OVRR, ECVRR, and ETVRR showed no prognostic impact on survival outcome (all $p>0.05$ ). To reveal most effective cutoff value of $V R R$, we assessed every 5\% interval around median. By this process, we concluded that ETVRR with 45\% cutoff value correlated significantly with OS $(p<0.0001)$ and LRPFS $(p=0.009)$ (Table 3). The median OS and LRPFS were 31.3 and 47.5 months for patients with greater than 45\% ETVRR, respectively, compared with 8.3 and 6.3 months for those with a lower than 45\% value (Fig. 2). Nodal status was a statistically significant prognostic factor of DMFS when comparing the N3 group versus NO-2 group $(p=0.049)$. Other factors of age, sex, ECOG PS, stage except for nodal status, RT dose, SER, PCI did not show statistically significant difference with regard to survival outcomes.

Because ETVRR was the only significant prognostic factor in the univariate analysis, we assessed the backward stepwise multivariate analysis and ETVRR was also shown as the only statistically significant factor for OS (HR, 0.117; $95 \% \mathrm{Cl}, 0.026$ to $0.530 ; p=0.005)$ and LRPFS (HR, $0.064 ; 95 \% \mathrm{Cl}, 0.007$ to $0.601 ; p=0.016$ ) (Table 4). 
Table 3. Univariate analysis of prognostic factors

\begin{tabular}{|c|c|c|c|c|c|c|c|c|c|}
\hline Variable & No. & $2-y r$ OS (\%) & $p$-value & 2-yr PFS (\%) & $p$-value & 2-yr LRPFS (\%) & $p$-value & $2-y r$ DMFS (\%) & $p$-value \\
\hline \multicolumn{10}{|l|}{ Age (yr) } \\
\hline$\leq 70$ & 29 & 46.5 & 0.274 & 30.4 & 0.232 & 64.7 & 0.048 & 69.3 & 0.279 \\
\hline$>70$ & 18 & 72.2 & & 44 & & 88.1 & & 70.5 & \\
\hline \multicolumn{10}{|l|}{ Sex } \\
\hline Male & 42 & 57.1 & 0.484 & 50.1 & 0.911 & 73.6 & 0.748 & 70.1 & 0.725 \\
\hline Female & 5 & 50 & & 53.3 & & 80 & & 66.7 & \\
\hline \multicolumn{10}{|l|}{ ECOG PS } \\
\hline$<2$ & 41 & 60 & 0.083 & 52.8 & 0.669 & 76.4 & 0.72 & 69.6 & 0.786 \\
\hline$\geq 2$ & 6 & 33.3 & & 25 & & 50 & & 66.7 & \\
\hline \multicolumn{10}{|l|}{ T stage } \\
\hline $1-2$ & 22 & 57.3 & 0.483 & 55.2 & 0.999 & 70.6 & 0.311 & 76.7 & 0.389 \\
\hline 3-4 & 25 & 56 & & 46.7 & & 77.8 & & 64.3 & \\
\hline \multicolumn{10}{|l|}{ N stage } \\
\hline $0-2$ & 32 & 64.6 & 0.203 & 55.5 & 0.548 & 73.4 & 0.625 & 77.8 & 0.049 \\
\hline 3 & 15 & 40 & & 39.1 & & 73.3 & & 53.9 & \\
\hline \multicolumn{10}{|l|}{ Stage } \\
\hline$|-||| A$ & 24 & 65.3 & 0.539 & 57.6 & 0.732 & 72.4 & 0.479 & 84.2 & 0.135 \\
\hline IIIB & 23 & 47.8 & & 43.7 & & 75.8 & & 56.3 & \\
\hline \multicolumn{10}{|l|}{ RT dose (Gy) } \\
\hline$\leq 54$ & 32 & 61.3 & 0.195 & 47.4 & 0.825 & 73.4 & 0.82 & 65 & 0.968 \\
\hline$>54$ & 15 & 46.7 & & 61.8 & & 76.6 & & 84 & \\
\hline \multicolumn{10}{|l|}{ SER (day) } \\
\hline$\leq 64$ & 24 & 56.5 & 0.754 & 54 & 0.614 & 72.2 & 0.58 & 71.8 & 0.846 \\
\hline$>64$ & 23 & 56.5 & & 46.6 & & 71 & & 68.8 & \\
\hline \multicolumn{10}{|l|}{$\mathrm{PCl}$} \\
\hline Yes & 38 & 54.1 & 0.317 & 52.8 & 0.22 & 75.7 & 0.446 & 74.4 & 0.168 \\
\hline No & 9 & 66.7 & & 40 & & 85.7 & & 53.3 & \\
\hline \multicolumn{10}{|c|}{ Initial GTV (mL) } \\
\hline$\leq 90$ & 24 & 61 & 0.73 & 46.6 & 0.225 & 74 & 0.686 & 62.3 & 0.077 \\
\hline$>90$ & 23 & 52.2 & & 54.9 & & 74.2 & & 77.6 & \\
\hline \multicolumn{10}{|l|}{ OVRR (\%) } \\
\hline$\leq 80$ & 20 & 50 & 0.667 & 38.8 & 0.439 & 62.2 & 0.564 & 64.9 & 0.87 \\
\hline$>80$ & 27 & 61.5 & & 59.1 & & 82.7 & & 74.2 & \\
\hline \multicolumn{10}{|l|}{ ECVRR (\%) } \\
\hline$\leq 50$ & 25 & 44 & 0.161 & 38.2 & 0.551 & 67.3 & 0.842 & 60.6 & 0.243 \\
\hline$>50$ & 22 & 71.4 & & 62.2 & & 81.3 & & 79.4 & \\
\hline \multicolumn{10}{|l|}{ ETVRR (\%) } \\
\hline$\leq 45$ & 3 & 0 & $<0.0001$ & 0 & 0.138 & 0 & 0.009 & 0 & 0.556 \\
\hline$>45$ & 27 & 61.5 & & 44.7 & & 70.3 & & 73.9 & \\
\hline
\end{tabular}

OS, overall survival; PFS, progression-free survival; LRPFS, locoregional progression-free survival; DMFS, distant metastasis-free survival; ECOG PS, Eastern Cooperative Oncology Group performance status; RT, radiotherapy; SER, start of any treatment to the end of radiation therapy; $\mathrm{PCl}$, prophylactic cranial irradiation; GTV, gross tumor volume; OVRR, overall volume reduction rate; ECVRR, early chemotherapy volume reduction rate; ETVRR, early treatment volume reduction rate.

We additionally analyzed the relationship between the time interval from first chemotherapy to first RT and ETVRR. Patients were divided into three groups depend on the timing of RT start. Group $1(n=8)$ and group $2(n=35)$ started RT with 1st and 2nd cycle of chemotherapy, and group $3(n=4)$ started RT with 3rd or thereafter. Median ETVRR of each group was 70.5\%, 76.9\%, and 66.7\%, respectively, and there was no statistical difference between the time interval and ETVRR ( $p=$ 0.619). 
A

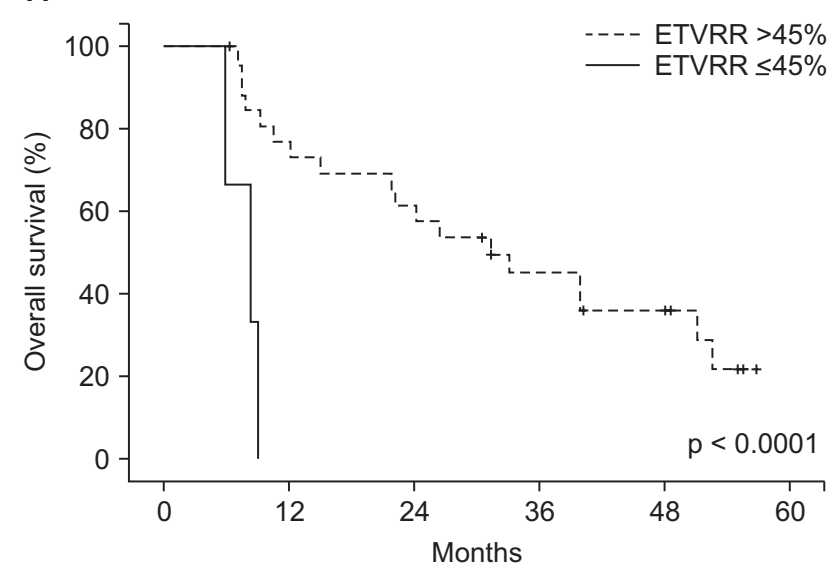

B

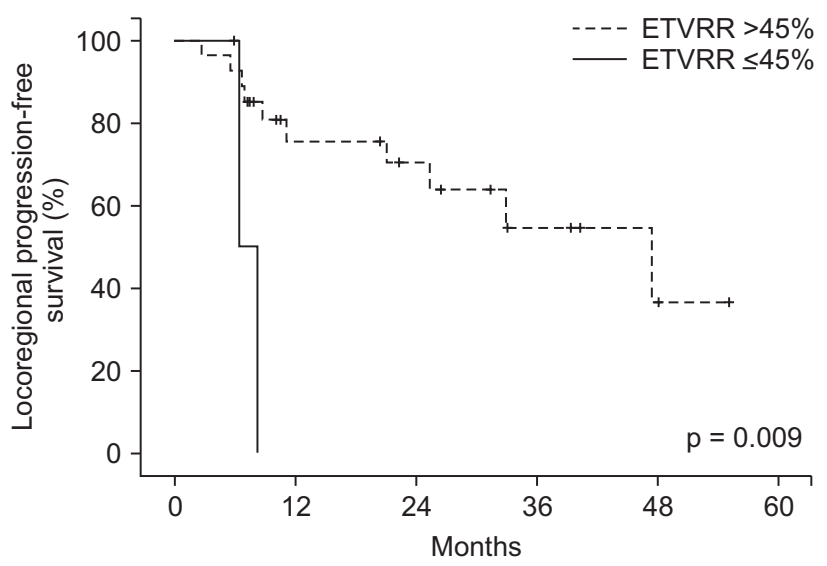

Fig. 2. Results based on early treatment volume reduction rate (ETVRR): (A) overall survival and (B) locoregional progression-free survival.

Table 4. Multivariate logistical regression analyses of prognostic factors

\begin{tabular}{|c|c|c|c|c|}
\hline \multirow{2}{*}{ Variable } & \multicolumn{2}{|c|}{ OS } & \multicolumn{2}{|c|}{ LRPFS } \\
\hline & HR $(95 \% \mathrm{Cl})$ & $p$-value & HR $(95 \% \mathrm{Cl})$ & $p$-value \\
\hline Age (>70 yr vs. $\leq 70$ yr) & - & - & $0.226(0.048-1.072)$ & 0.061 \\
\hline Sex (male vs. female) & $0.27(0.034-2.126)$ & 0.214 & $6.009(0.502-71.951)$ & 0.157 \\
\hline N stage (0-2 vs. 3) & - & - & $0.207(0.023-1.860)$ & 0.160 \\
\hline ETVRR ( $>45 \%$ vs. $\leq 45 \%)$ & $0.117(0.026-0.530)$ & 0.005 & $0.064(0.007-0.601)$ & 0.016 \\
\hline
\end{tabular}

OS, overall survival; LRPFS, locoregional progression-free survival; HR, hazard ratio; $\mathrm{Cl}$, confidence interval; ETVRR, early treatment volume reduction rate.

\section{Discussion and Conclusion}

The aim of this study was to determine prognostic factors influencing CRT outcome for LS-SCLC, including tumor volume parameters such as OVRR, ECVRR and ETVRR. We hypothesized that initial GTV and VRR of gross tumor are prognostic factors related to patient survival. The median OVRR was $81.6 \%$, showing substantial GTV reduction within three months after RT; however, an OVRR $>80 \%$ was not a statistically significant prognostic factor of any survival outcome. The median ETVRR was $71.4 \%$ (range, 30\% to 97.6\%), which showed a substantial degree of GTV reduction during early CRT, reflecting the radiosensitivity of SCLC. An ETVRR $>45 \%$ was a significant prognostic factor of OS $(p<0.0001)$ and LRPFS $(p=0.009)$.

Tumor volume defined in CT images is a main concept of 3DCRT. Before the CT era, the largest longitudinal diameter was the only detectable prognostic factor related to tumor size. In RTOG 73-01 [16], tumor diameter measurement was based on posterior-anterior and lateral chest radiography. With this two-dimensional assessment, 91\% of patients treated with $60 \mathrm{~Gy}$ showed complete response, partial response or stable disease status and only 9\% had a progressive response. Such favorable outcomes differ considerably from the outcomes of more recent studies of lung cancer treatment. To compensate for this over-estimation, the definition of $\mathrm{CR}$ was changed to include radiographic, clinical, and bronchoscopic results $[17,18]$. However, these methods are still less objective than imageonly method due to inter-observer variation. The AJCC TNM staging system [13], one of most widely accepted prognostic factors of lung cancer, also uses greatest tumor dimension based on CT images for T staging.

After the introduction of 3DCRT for cancer treatment, physicians began to contour the tumor shape on each planning CT slice. Tumor volume was considered as a possible prognostic factor based on clinical experiences. Volumetric tumor measurement was also considered more objective and accurate than clinical evaluation of tumor response.

There have been several studies regarding initial tumor volume as a prognostic factor in lung cancer. Bradley et al. [19] demonstrated that GTV was a prognostic factor for OS and cause-specific survival in NSCLC treated with 3DCRT planning. In that study, $\mathrm{T}, \mathrm{N}$, and overall stage were not 
statistically significant prognostic factors. Werner-Wasik et al. [20] investigated the efficacy of initial GTV for NSCLC patients in the RTOG 93-11 phase I-II trial. In their doseescalation study, patients with tumor volume smaller than 45 $\mathrm{cm}^{3}$ achieved better median survival time and PFS compared to those of patients with larger tumors. Basaki et al. [21] also showed that primary tumor volume had an impact on OS in 71 NSCLC stage III patients treated with definitive CRT ( $n=$ 45 ) or RT alone ( $n=26$ ). In that study, GTV and total tumor volume were statistically meaningful, but nodal volume alone was not. Koo et al. [22] conducted a single-institution study that retrospectively reviewed 191 patients with stage III NSCLC who underwent CRT. They concluded that smaller pretreatment GTV is an independent prognostic factor of better OS, PFS, and LRPFS (all $p<0.05$ ). They also studied the impact of VRR as a prognostic factor of survival outcome; however, it showed only a trend of OS ( $p=0.075)$. Reymen et al. [8] revealed total GTV, including gross tumor with selective LNs to be irradiated, as an independent prognostic factor of overall survival in stage I to III SCLC. This was the first study to show the prognostic significance of total GTV in SCLC. Initial GTV was not a prognostic factor in this study, and OVRR was also not statistically significant. We hypothesize that these results are due to the small number of patients and the fact that SCLC has a favorable treatment response, as reported in previous studies [23], so almost every patient experienced substantial volume reduction at follow-up CT.

A limited number of studies have concentrated on the rate of 3D volume reduction. VRR was studied in head and neck cancer by Yang et al. [10] and was an effective predictor of local control in oropharynx and hypopharynx cancer patients treated with IMRT. Therefore, it was recommended that patients who showed a less than 50\% volume reduction should be considered for other treatment options like salvage surgery or escalated dose prescription. In 2001, Werner-Wasik et al. [11] reported that VRR was an effective prognostic factor of response evaluation in locally-advanced NSCLC patients. Since then, however, no studies have produced meaningful results regarding volume changes in lung cancer. This study was the first attempt to reveal the prognostic importance of VRR in SCLC.

The ETVRR $<45 \%$ group patients showed poor OS and LRPFS (8.3 and 6.3 months, respectively) and this was relatively poor survival outcome than previous studies. The reason was because of early locoregional failure in ipsilateral lung parenchyme $(n=2)$ and brain metastasis $(n=1)$.
This study has some limitations. Established prognostic factors in SCLC (age, sex, ECOG PS, SER, initial GTV, and overall treatment response) were not significantly correlated with survival outcome in this study, which may have been influenced by the relatively small number of patients included in analysis. Not all patients were subjected to adaptive planning, and ETVRR was determined in only 30 of 47 patients. Currently, most SCLC patients treated with CRT at our institution undergo adaptive planning; a longer follow-up with a larger number of patients will reveal the validity of these results.

In conclusion, overall and early treatment responses to chemo-radiation in LS-SCLC as measured by volume reduction were favorable. Patients who showed an early treatment response (ETVRR >45\%) had better treatment outcome in terms of PFS and OS than those who did not. ETVRR as a parameter for early treatment response may be a useful prognostic factor of treatment outcome in LS-SCLC patients treated with CRT.

\section{Conflict of Interest}

No potential conflict of interest relevant to this article was reported.

\section{References}

1. Adjei AA, Marks RS, Bonner JA. Current guidelines for the management of small cell lung cancer. Mayo Clin Proc 1999; 74:809-16.

2. Siegel R, Ward E, Brawley O, Jemal A. Cancer statistics, 2011: the impact of eliminating socioeconomic and racial disparities on premature cancer deaths. CA Cancer J Clin 2011;61:212-36.

3. Warde $P$, Payne D. Does thoracic irradiation improve survival and local control in limited-stage small-cell carcinoma of the lung? A meta-analysis. J Clin Oncol 1992;10:890-5.

4. Pignon JP, Arriagada $R$, Ihde $D C$, et al. A meta-analysis of thoracic radiotherapy for small-cell lung cancer. $N$ Engl J Med 1992;327:1618-24.

5. van Meerbeeck JP, Fennell DA, De Ruysscher DK. Small-cell lung cancer. Lancet 2011;378:1741-55.

6. International Commission on Radiation Units and Measurements. Prescribing, recording, and reporting photon beam therapy (Report 50). Bethesda, MD: International Commission on Radiation Units and Measurements; 1993.

7. Dehing-Oberije $C, Y u$ S, De Ruysscher D, et al. Development and external validation of prognostic model for 2-year 
survival of non-small-cell lung cancer patients treated with chemoradiotherapy. Int J Radiat Oncol Biol Phys 2009;74:35562.

8. Reymen B, Van Loon J, van Baardwijk et al. Total gross tumor volume is an independent prognostic factor in patients treated with selective nodal irradiation for stage I to III small cell lung cancer. Int J Radiat Oncol Biol Phys 2013;85:1319-24.

9. Kim YH, Kim DY, Kim TH, et al. Usefulness of magnetic resonance volumetric evaluation in predicting response to preoperative concurrent chemoradiotherapy in patients with resectable rectal cancer. Int J Radiat Oncol Biol Phys 2005;62:761-8.

10. Yang SN, Liao CY, Chen SW, et al. Clinical implications of the tumor volume reduction rate in head-and-neck cancer during definitive intensity-modulated radiotherapy for organ preservation. Int J Radiat Oncol Biol Phys 2011;79:1096-103.

11. Werner-Wasik M, Xiao Y, Pequignot E, Curran WJ, Hauck $W$. Assessment of lung cancer response after nonoperative therapy: tumor diameter, bidimensional product, and volume: a serial CT scan-based study. Int J Radiat Oncol Biol Phys 2001;51:56-61.

12. Micke $P$, Faldum $A$, Metz $T$, et al. Staging small cell lung cancer: Veterans Administration Lung Study Group versus International Association for the Study of Lung Cancer: what limits limited disease? Lung Cancer 2002;37:271-6.

13. Sobin LH, Compton CC. TNM seventh edition: what's new, what's changed: communication from the International Union Against Cancer and the American Joint Committee on Cancer. Cancer 2010;116:5336-9.

14. Eisenhauer EA, Therasse $P$, Bogaerts J, et al. New response evaluation criteria in solid tumours: revised RECIST guideline (version 1.1). Eur J Cancer 2009;45:228-47.

15. Cockcroft DW, Gault MH. Prediction of creatinine clearance from serum creatinine. Nephron 1976;16:31-41.

16. Perez $C A$, Stanley $K$, Rubin $P$, et al. A prospective randomized study of various irradiation doses and fractionation schedules in the treatment of inoperable non-oat-cell carcinoma of the lung: preliminary report by the Radiation Therapy Oncology Group. Cancer 1980;45:2744-53.

17. Le Chevalier T, Arriagada R, Quoix E, et al. Radiotherapy alone versus combined chemotherapy and radiotherapy in nonresectable non-small-cell lung cancer: first analysis of a randomized trial in 353 patients. J Natl Cancer Inst 1991;83: 417-23.

18. Le Chevalier T, Arriagada $R$, Tarayre M, et al. Significant effect of adjuvant chemotherapy on survival in locally advanced non-small-cell lung carcinoma. J Natl Cancer Inst 1992;84:58.

19. Bradley JD, leumwananonthachai N, Purdy JA, et al. Gross tumor volume, critical prognostic factor in patients treated with three-dimensional conformal radiation therapy for nonsmall-cell lung carcinoma. Int J Radiat Oncol Biol Phys 2002; 52:49-57.

20. Werner-Wasik M, Swann RS, Bradley J, et al. Increasing tumor volume is predictive of poor overall and progression-free survival: secondary analysis of the Radiation Therapy Oncology Group 93-11 phase I-II radiation dose-escalation study in patients with inoperable non-small-cell lung cancer. Int J Radiat Oncol Biol Phys 2008;70:385-90.

21. Basaki K, Abe $Y$, Aoki M, Kondo H, Hatayama Y, Nakaji S. Prognostic factors for survival in stage III non-small-cell lung cancer treated with definitive radiation therapy: impact of tumor volume. Int J Radiat Oncol Biol Phys 2006;64:449-54.

22. Koo TR, Moon SH, Lim YJ, et al. The effect of tumor volume and its change on survival in stage III non-small cell lung cancer treated with definitive concurrent chemoradiotherapy. Radiat Oncol 2014;9:283.

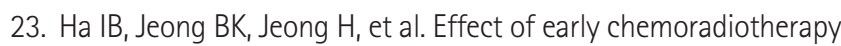
in patients with limited stage small cell lung cancer. Radiat Oncol J 2013;31:185-90. 